\title{
Direct probe of Mott-Hubbard to charge-transfer insulator transition and electronic structure evolution in transition-metal systems
}

\author{
P. Olalde-Velasco ${ }^{1,2}$, J. Jiménez-Mier ${ }^{2 *}$, J.D. Denlinger ${ }^{1}$, Z. Hussain ${ }^{1}$, W.L. Yang ${ }^{1 \dagger}$ \\ ${ }^{1}$ Advanced Light Source, Lawrence Berkeley National Laboratory, Berkeley, California \\ 94720, USA \\ ${ }^{2}$ Instituto de Ciencias Nucleares, UNAM, 04510 Mexico DF, Mexico \\ (Received ; Published )
}

We report the most direct experimental verification of Mott-Hubbard and Charge-Transfer insulators through X-ray emission spectroscopy in transition-metal fluorides. The $p$ - $d$ hybridization features in the spectra allow a straightforward energy alignment of the anion- $2 p$ and metal-3d valence states, which visually shows the difference between the two types of insulators. Further, in parallel with the theoretical Zaanen-Sawatzky-Allen diagram, a complete experimental systematics of the $3 d$ Coulomb interaction and $2 p-3 d$ charge-transfer energy is reported, and could serve as a universal experimental trend for other transition-metal systems including oxides.

PACS numbers: 71.20.-b, 71.27.+a, 78.70.En, 78.70.Dm 
The emergent phenomena discovered in $3 d$ transition-metal compounds, such as cuprates, manganites, cobaltates, and iron pnictides, have revolutionized our understanding of both fundamental physics and practical materials. In 1985, the seminal work of Zaanen-Sawatzky-Allen (ZSA) diagram ${ }^{1}$ was formulated to categorize the transition-metal compounds. According to this diagram ${ }^{1-3}$, both the Coulomb interaction of the transition-metal (TM) $3 d$ electrons, $\mathrm{U}_{d d}$, and the charge-transfer energy between TM- $3 d$ and anion- $p$ states, $\Delta_{\mathrm{CT}}$, are important parameters to regulate the fundamental electronic structure. Two types of insulators, namely Mott-Hubbard (M-H) and charge-transfer (C-T) insulators, are defined by comparing the value of $\mathrm{U}_{d d}$ with $\Delta_{\mathrm{CT}}$ (Fig.1). Ever since, intensive experimental efforts have focused on deducing these key parameters ${ }^{2,4-21}$; however, a direct experimental result of a complete series of $\mathrm{U}_{d d}$ and $\Delta_{\mathrm{CT}}$ is still unavailable. Here we present a systematic X-ray spectroscopic study on both the occupied and unoccupied states of a series of TM difluorides $\left(\mathrm{MF}_{2}\right)$. The features from $d-p$ hybridization enable a simple and self-consistent energy alignment through hybridization (EATH) for plotting TM-3d and anion- $p$ states on a common energy scale, revealing directly the different type of insulators. With the data on both valence and conduction states, a complete and absolute experimental systematics of $\mathrm{U}_{\mathrm{dd}}$ and $\Delta_{\mathrm{CT}}$ is further reported, in parallel with the theoretical ZSA diagram. The significance of the ZSA diagram is that the valence states in the vicinity of the Fermi level could be from not only the TM-3d, but the anion- $p$ states (Fig.1). This was later found to be the case for superconducting cuprates, and has been providing an important foundation for studying $3 d$ TM systems (e.g. ${ }^{22}$ ). Furthermore, the relative energy position of TM-3d and anion-p states in TM compounds has been one of the most important electronic structures that define the performance of various devices like organic solar cells and lithium batteries ${ }^{23}$. 
Therefore, such a direct experimental probe is of both fundamental and technological importance, which has not been achieved due to the great challenges on distinguishing the density of states (DOS) with elemental (anion vs TM) and orbital ( $2 p$ vs $3 d$ ) selectivity as well as determining the relative energy positioning of TM-3d and anion-p states.

Previous studies of ZSA diagram in TM oxides and halides are mostly based on photoelectron spectroscopy coupled with theory, e.g., lineshape of core-level photoelectron ${ }^{4-9}$, valence band photoelectron ${ }^{9-12}$, and resonant photoemission ${ }^{9,13-17}$ spectroscopy. Photoelectron spectroscopy probes total DOS, which inherently requires theoretical aid for resolving the TM $3 d$-DOS and anion $2 p$-DOS. The angle resolved photoemission ${ }^{18}$ is limited by the availability of conductive and cleavable single crystal samples. Additionally, with few experimental results of inverse photoemission on $\mathrm{NiO}$ and $\mathrm{MnO}^{3,19,20}$, photoemission probes only occupied states thus cannot provide direct experimental values of either $U_{d d}$ or $\Delta_{\mathrm{CT}}$, because of the missing information on the energy levels of unoccupied states.

X-ray absorption spectroscopy (XAS) and X-ray emission spectroscopy (XES) are bulk probes measuring both unoccupied and occupied states with the inherent elemental and orbital selectivity ${ }^{4}$. Previously, Ni- $L^{21}$, Oxygen-K ${ }^{24,25}$ and Fluorine-K ${ }^{26-28}$ edges were studied by either XAS or XES. However, the energy values of the spectra are in respect of the core levels of the particular elements. A complete experimental picture on the Mott-Hubbard (M-H) to Charge-Transfer (C-T) transition, as well as the evolution of $\mathrm{U}_{d d}$ and $\Delta_{\mathrm{CT}}$, has never been achieved due to the lack of the method for obtaining the relative energy position of the $d$ and $\mathrm{p}$ states, and the difficulty in choosing an appropriate system.

The system we chose is $3 d$ transition-metal difluorides, $\mathrm{MF}_{2}$ with $\mathrm{M}=\mathrm{Cr}-\mathrm{Zn}$. Our data show that $\mathrm{MF}_{2}$ is a cleaner system for testing conceptual issues than oxides due to their 
extreme ionic status ${ }^{29}$. Additionally, $\mathrm{MF}_{2}$ was only marginally studied ${ }^{8,12,26-28,30}$. There are still controversial questions on the type of insulators for the higher members in the series 2,8 , ${ }^{30}$, on the understanding of the Fluorine XES lineshape ${ }^{26,28,31}$, and on the irregular variation of the leading edges of XAS spectra ${ }^{26,27}$. All these questions are naturally answered in this letter.

$\mathrm{MF}_{2}$ samples were purchased from Sigma-Aldrich. Spectroscopic measurements were performed at Beamline 8.0.1 at the ALS. Experiments were done at room temperature and with the linear polarization of the incident beam $45^{\circ}$ to sample surfaces. The XAS spectra were collected in the total electron yield (TEY) mode by registering the sample current normalized to the photon flux. The XES spectra were obtained under non-resonant condition ${ }^{4}$ with excitation energies of 600, 660, 730, 800, 880, $968.91094 .1 \mathrm{eV}$ for Cr-, Mn-, Fe-, Co-, $\mathrm{Ni}-, \mathrm{Cu}-, \mathrm{Zn}-L$ edges. The resolution for XAS and XES measurements are $0.2 \mathrm{eV}$ and $0.7 \mathrm{eV}$ respectively. All F-K spectra were collected in one experiment, with $700 \mathrm{eV}$ excitation energy for XES measurements, to keep the relative energy shift reliable.

Our central result on the crossover of $\mathrm{F}-2 p$ and $\mathrm{TM}-3 d$ valence states in $\mathrm{MF}_{2}$ system is depicted in Fig.2a. Valence states from F-2 $p$ and TM-3d were measured through XES of Fluorine $\mathrm{K}_{\alpha}(\mathrm{F}-\mathrm{K})$ and $\mathrm{TM} \mathrm{L}_{\alpha}(\mathrm{M}-\mathrm{L})$ respectively ${ }^{4}$. Because only relative energy shifts are of interest here, the Fermi level was arbitrarily set at the leading edge of the spectrum of $\mathrm{CrF}_{2}$. For F- $2 p$ states, the main peak position is almost stable from $\mathrm{CrF}_{2}$ to $\mathrm{ZnF}_{2}$. The most systematic change of the F-K lineshape is on the high energy shoulders at the $-4 \mathrm{eV}$ to $0 \mathrm{eV}$ energy range, which are obvious in a magnified view (Fig.2b-d, and Fig.S1 ${ }^{29}$ ). From $\mathrm{CrF}_{2}$ to $\mathrm{CuF}_{2}$, these shoulders shift towards the main peak with intensity getting stronger mainly due to the closer position to the main peak. Nevertheless, for $\mathrm{ZnF}_{2}$, this shoulder feature 
disappears and a hump develops on the low energy side of the main F-K peak, while a high energy shoulder in M-L emerges simultaneously.

The shoulder and hump features in $\mathrm{MF}_{2}$ system, much clearer than that in oxides ${ }^{25,29}$, enables a straightforward way to align M-L with F-K XES spectra on a common energy scale, so the following conditions are met simultaneously for each component: (i) the leading edges of M-L are aligned to the leading edges of the F-K spectra; (ii) shoulders in F-K are aligned with peaks of M-L spectra; (iii) shoulders in M-L are aligned with peaks of F-K spectra. The consistency between the three types of alignments strongly suggests the validity of this energy alignment. For example, in Fig.2b, if the low (high) energy shoulder of F-K (M-L) in $\mathrm{ZnF}_{2}$ is aligned with the main peak of M-L (F-K), the leading edges of F-K and M-L will be aligned simultaneously.

While the detailed discussion on the XES lineshape is very complicated and beyond the scope of this work, we focus on the energy position of the lower Hubbard bands (LHBs) that are defined by the top TM-3d valence states revealed by the highest energy peak in M-L XES (bars in Fig.2a). The LHB is located on the higher energy side of the corresponding F- $2 p$ state for $\mathrm{CrF}_{2}$ to $\mathrm{CuF}_{2}$ (M-H insulators), but evolves towards lower energy and eventually merges into the wide $\mathrm{F}-2 p$ band for $\mathrm{ZnF}_{2}$ (intermediate or C-T insulator). The consistency on the systematic evolution between the F-K shoulders and M-L leading peak position (LHB) confirms that the shoulder and hump features originate from $3 d-2 p$ hybridization. This suggests the self-consistence of the EATH method and is in line with the schematic in Fig.1. In order to obtain a complete experimental picture on both the occupied and unoccupied states around Fermi level, thus $\mathrm{U}_{d d}$ and $\Delta_{\mathrm{CT}}$, we combined XAS of F-K edges with XES data in Fig.3. Our XAS spectra are consistent with the recent study on F-K XAS in difluorides ${ }^{27}$. 
Note that the band gap is indicated by the leading edges of XAS and XES, which is not a topic of this report. Here we concentrate on the evolution of the highest energy F- $2 p$ top valence states (black bars), LHB states (red or gray bars, from TM-3d in Fig.2a), and upper Hubbard band (UHB) states defined by the lowest energy peaks in XAS (dashed bars).

Fig.4 shows the summary of the experimental $\mathrm{U}_{d d}$ and $\Delta_{\mathrm{CT}}$ in $\mathrm{MF}_{2}$ derived from Fig.3, with values presented in Table $\mathrm{S} 1$ of ref. ${ }^{29} . \mathrm{U}_{d d},\left(\Delta_{\mathrm{CT}}\right)$ is set as the energy difference between the red/gray (black) and dashed bars in Fig.3, which corresponds to the theoretical energy value of the Hund's rule ground state term of $3 d^{\mathrm{x}}$ configurations $^{2}$. For direct comparison, we also plotted the theoretical values from Zaanen-Sawatzky (ZS) analysis ${ }^{22}$. The theoretical $\Delta_{\mathrm{CT}}$ for oxides was increased by $3 \mathrm{eV}$ to compensate the difference on electronegativity between $\mathrm{F}$ and $\mathrm{O}$, a method recommended by the original ZS analysis ${ }^{2}$. The most important physics is on the trend of the evolution ${ }^{2}$. The experimental and theoretical values of $\mathrm{U}_{d d}$ and $\Delta_{\mathrm{CT}}$ share the same trend from $\mathrm{CrF}_{2}$ to $\mathrm{CuF}_{2}$ (Fig.4) except for $\mathrm{FeF}_{2}$. The $\mathrm{FeF}_{2}$ may contain contamination of $\mathrm{Fe}^{3+}$ as indicated by the XAS lineshape ${ }^{24}$; however, the much lower experimental $\Delta_{\mathrm{CT}}$ of $\mathrm{FeF}_{2}$ cannot be simply attributed to contamination, because $\mathrm{Fe}^{3+}$ should display a much higher value of $\Delta_{\mathrm{CT}}$ as for $\mathrm{MnF}_{2}$. This qualitative discrepancy calls for theoretical revisit into the $\mathrm{Fe}^{2+}$ compound.

With the agreement between our experimental results and the theoretical ZS analysis ${ }^{2}$, we reached a complete understanding on the electronic structure evolution of TM compounds. We first describe the occupied states through XES data. When we advance from lighter to heavier TM elements, the increasing nuclear charge enhances the binding energy of $3 d$ electrons, leading to a monotonic decrease on M-L XES energy (Fig.2). For the anion-2p states, $\mathrm{F}-2 p$ here, the system is getting more covalent from $\mathrm{CrF}_{2}$ to $\mathrm{CuF}_{2}$, thus less ionic and 
lower in binding energy. This effect is very small though because $\mathrm{MF}_{2}$ remains a strong ionic system. The effect is exhibited by the broadening of the F-K XES main peak, and the monotonic but small $(<1 \mathrm{eV})$ increase in XES energy (Fig.2).

Secondly, contrasting the monotonic evolution of the occupied states in XES, the unoccupied states have to vary a great amount (Fig.3) to accommodate the big changes on $\mathrm{U}_{d d}$ and $\Delta_{\mathrm{CT}}$ (Fig.4). In general, the spatial extent of $d$ orbitals shrinks with atomic number, leading to a trend of increasing $\mathrm{U}_{d d}$. However, $\mathrm{MnF}_{2}$ is a half-filled $d^{5}$ system, therefore, to add an electron to a high spin $d^{5}$ states means it has to go in with spin down, thus there is no energy gain from the exchange. Such exchange stabilization leads to a sharp maximum at $\mathrm{Mn}^{2+}$ on both $\mathrm{U}_{d d}$ and $\Delta_{\mathrm{CT}}{ }^{19}$ (Fig.4). The decreasing $\mathrm{U}_{d d}$ from $\mathrm{NiF}_{2}$ to $\mathrm{CuF}_{2}$ is attributed to the spatially expanded $d$ orbitals with increasing atomic radii from $\mathrm{Ni}(1.25 \AA)$ to $\mathrm{Cu}(1.28 \AA)$, which is due to the stronger electron-electron repulsion than the effect from increasing nuclear charges.

$\mathrm{ZnF}_{2}$ is a special component in the $\mathrm{MF}_{2}$ system. The $3 d$ shell is full for $\mathrm{Zn}^{2+}$, so there is no UHB. Our XES data (Fig.2) show that the electron removal Zn-3d state is either merged into the broad F- $2 p$ bands (red bar) or deep below it (dotted bar), and only broad features corresponding to $\mathrm{Zn}-4 s, 4 p$ hybridized with F- $2 p$ are observable in XAS (Fig.3). Therefore, the first electron removal state is of F- $2 p$ character, and the first electron addition state is of $\mathrm{Zn}-4 s, 4 p$, a charge excitation from valence to conduction band involves a charge transfer process, $\mathrm{ZnF}_{2}$ is thus a $\mathrm{C}-\mathrm{T}$ insulator with $\Delta_{\mathrm{CT}}$ of about $8.3 \mathrm{eV}$.

The reason that almost all $\mathrm{MF}_{2}$ samples are $\mathrm{M}-\mathrm{H}$ insulators is because fluorine is the most electronegative element, resulting in the lowest $2 p$ level and the maximum charge transfer energy (Fig.4c). By a simple reduction of our experimental $\Delta_{\mathrm{CT}}$ by $3 \mathrm{eV}$ to compensate the 
difference on electronegativity between $\mathrm{F}$ and $\mathrm{O}^{2}$, a qualitative trend of $\Delta_{\mathrm{CT}}$ for TM oxides is generated. The derived $\Delta_{\mathrm{CT}}$, comparing with the experimental $\mathrm{U}_{\mathrm{dd}}$, successfully displays a $\mathrm{M}-\mathrm{H}$ to $\mathrm{C}-\mathrm{T}$ insulator transition between $\mathrm{CoO}$ and $\mathrm{NiO}$, leaving $\mathrm{CuO}$ an obvious $\mathrm{C}-\mathrm{T}$ insulator (Fig.4c).

In summary, this work provided the first direct experimental probe of $\mathrm{M}-\mathrm{H}$ and $\mathrm{C}-\mathrm{T}$ insulators, and the first pure experimental verification of ZSA diagram. The reported experimental systematics of $\mathrm{U}_{\mathrm{dd}}$ and $\Delta_{\mathrm{CT}}$ could serve as a general trend for other TM compounds. More importantly, the demonstrated EATH method will empower researchers to probe the relative energy position of TM- $d$ and anion- $p$ states, which is critical in various fields of material sciences.

\section{Acknowledgements}

We thank G.A. Sawatzky for his insight on data interpretation. The ALS at LBNL is supported by the U.S. DOE under Contract No. DE-AC02-05CH11231. Work in Mexico is supported by grants UNAM-PAPIIT IN109308, CONACYT U41007-F CONACyT 56764. PO and JJ thank the support from CONACyT Mexico. WLY thanks the support from LDRD of LBNL.

*jimenez@nucleares.unam.mx

†wlyang@lbl.gov

${ }^{1}$ J. Zaanen, G. A. Sawatzky, and J. W. Allen, Physical Review Letters 55, 418 (1985).

${ }^{2}$ J. Zaanen and G. A. Sawatzky, Journal of solid state chemistry 88, 8 (1990). 
${ }^{3}$ J. Zaanen, G. A. Sawatzky, and J. W. Allen, Journal of Magnetism and Magnetic Materials 54-57, 607 (1986).

${ }^{4}$ F. de Groot and A. Kotani, Core Level Spectroscopy of Solids (CRC Press Taylor \& Francis Group, Boca Raton, 2008).

${ }^{5}$ A. E. Bocquet, T. Mizokawa, K. Morikawa, A. Fujimori, S. R. Barman, K. Maiti, D. D. Sarma, Y. Tokura, and M. Onoda, Physical Review B 53, 1161 (1996).

${ }^{6} \mathrm{~A}$. Kotani and K. Okada, Recent advances in magnetism of transition metal compounds (World Scientific Publishign Ltd, Singapore, 1993).

${ }^{7}$ G. Lee and S. J. Oh, Physical Review B 43, 14674 (1991).

${ }^{8}$ J. Park, S. Ryu, M.-s. Han, and S. J. Oh, Physical Review B 37, 10867 (1988).

${ }^{9}$ M. Imada, A. Fujimori, and Y. Tokura, Reviews of Modern Physics 70, 1039 (1998).

${ }^{10}$ R. T. Poole, J. A. Nicholson, J. Liesegang, J. G. Jenkin, and R. C. G. Leckey, Physical Review B 20, 1733 (1979).

${ }^{11}$ R. T. Poole, J. D. Riley, J. G. Jenkin, J. Liesegang, and R. C. G. Leckey, Physical Review B 13, 2620 (1976).

${ }^{12}$ S. P. Kowalczyk, L. Ley, F. R. McFeely, and D. A. Shirley, Physical Review B 15, 4997 (1977).

${ }^{13}$ A. Fujimori, et al., Physical Review B 42, 7580 (1990).

${ }^{14}$ A. Kakizaki, K. Sugeno, T. Ishii, H. Sugawara, I. Nagakura, and S. Shin, Physical Review B 28, 1026 (1983).

${ }^{15}$ A. Tanaka and T. Jo, Journal of the Physical Society of Japan 63, 2788 (1994).

${ }^{16}$ S. J. Oh, J. W. Allen, I. Lindau, and J. C. Mikkelsen, Physical Review B 26, 4845 (1982).

${ }^{17}$ M. R. Thuler, R. L. Benbow, and Z. Hurych, Physical Review B 27, 2082 (1983). 
${ }^{18}$ Z. X. Shen, C. K. Shih, O. Jepsen, W. E. Spicer, I. Lindau, and J. W. Allen, Physical

Review Letters 64, 2442 (1990).

${ }^{19}$ J. van Elp, R. H. Potze, H. Eskes, R. Berger, and G. A. Sawatzky, Physical Review B 44, 1530 (1991).

${ }^{20}$ G. A. Sawatzky and J. W. Allen, Physical Review Letters 53, 2339 (1984).

${ }^{21}$ G. van der Laan, J. Zaanen, G. A. Sawatzky, R. Karnatak, and J. M. Esteva, Physical Review B 33, 4253 (1986).

${ }^{22}$ C. Weber, K. Haule, and G. Kotliar, Nat Phys 6, 574 (2010).

${ }^{23}$ G.-A. Nazri and G. Pistoia, Lithium Batteries Science and Technology (Springer, New York, 2009).

${ }^{24}$ F. M. F. de Groot, M. Grioni, J. C. Fuggle, J. Ghijsen, G. A. Sawatzky, and H. Petersen, Physical Review B 40, 5715 (1989).

${ }^{25}$ E. Z. Kurmaev, R. G. Wilks, A. Moewes, L. D. Finkelstein, S. N. Shamin, and J. Kuneš, Physical Review B 77, 165127 (2008).

${ }^{26}$ C. Sugiura, Journal of the Physical Society of Japan 60, 2710 (1991).

${ }^{27}$ A. S. Vinogradov, et al., Physical Review B 71, 045127 (2005).

${ }^{28}$ E. I. Esmail and D. S. Urch, Spectrochimica Acta Part A: Molecular Spectroscopy 39, 573 (1983).

${ }^{29}$ See supplementary material at [URL will be inserted by AIP] for figures of fluorides and oxides, and values plotted in Fig.4.

${ }^{30}$ K. Okada and A. Kotani, Journal of the Physical Society of Japan 61, 4619 (1992).

${ }^{31}$ A. S. Koster, Journal of Physics and Chemistry of Solids 32, 2685 (1971). 


\section{Figure legends}

Fig.1 (Color online) Schematic of C-T (top) and M-H (bottom) insulators. The lower intensity $d$-p and $p$ - $d$ humps are hybridization features in $d$ - and $p$-partial density of states respectively. Such features are evident in $\mathrm{MF}_{2}$ system, enabling a straightforward alignment of the experimental partial DOS of TM-3d with F-2p.

Fig.2 (Color online) Experimental metal-3d and anion- $2 p$ valence states in $\mathrm{MF}_{2}$ aligned by EATH. (a) XES spectra of TM- $L \alpha$ in red (gray) and F- $K \alpha$ in blue (black) displays the partial $3 d$ - and $2 p$ - density of states respectively. The arbitrary intensity of the TM- $L \alpha$ and F-K $\alpha$ spectra are scaled to the same maximum value for each component. Bars are LHBs indicated by the highest energy TM- $3 d$ peaks from top valence states. From $\mathrm{CrF}_{2}$ to $\mathrm{CuF}_{2}$, TM-3d LHBs shifts towards lower energy, in parallel with the shrinking of the hybridization

shoulder in F-K $\alpha$ spectra ${ }^{29} \cdot \mathrm{ZnF}_{2}$ is the only C-T or intermediate insulator with $\mathrm{Zn}-3 d$ under F-2p valence states. (b)-(d) show three amplified examples on the energy alignments ${ }^{29}$. The leading edges of F- $K$ and TM- $L 3$, the emission peaks of TM- $L 3(\mathrm{~F}-K)$ and hybridization features of F- $K(\mathrm{TM}-L 3)$ are aligned respectively.

Fig.3 (Color online) Fluorine-K XES and XAS experimental data plotted on a common energy scale. Red (gray) bars indicate LHBs from Fig.2a. Black bars indicate the highest energy F- $2 p$ valence states. The TM- $3 d$ UHBs are defined by the lowest energy intensity peaks in XAS data, the so-called in-gap states (dashed bars). The data exhibit monotonic 
energy shift on occupied F-2p and LHB states contrasting very irregular energies of unoccupied UHBs.

Fig.4 (Color online) Systematics of $U_{d d}$ and $\Delta_{C T}$ generated from experiments on TM fluorides and their extension to oxides. (a) Experimental $U_{d d}$ from Fig.3 in comparison with theoretical Zaanen-Sawatzky analysis. (b) Experimental and theoretical $\Delta_{\mathrm{CT}}$ basically follow the same trend as that of $U_{d d}$. (c) Direct comparison of experimental $U_{d d}$ with $\Delta_{C T}$ in difluorides and oxides. $\Delta_{\mathrm{CT}}$ were decreased by $3 \mathrm{eV}$ for oxides to compensate the difference on electronegativity between $\mathrm{F}$ and $\mathrm{O}^{2}$. 
Fig.1

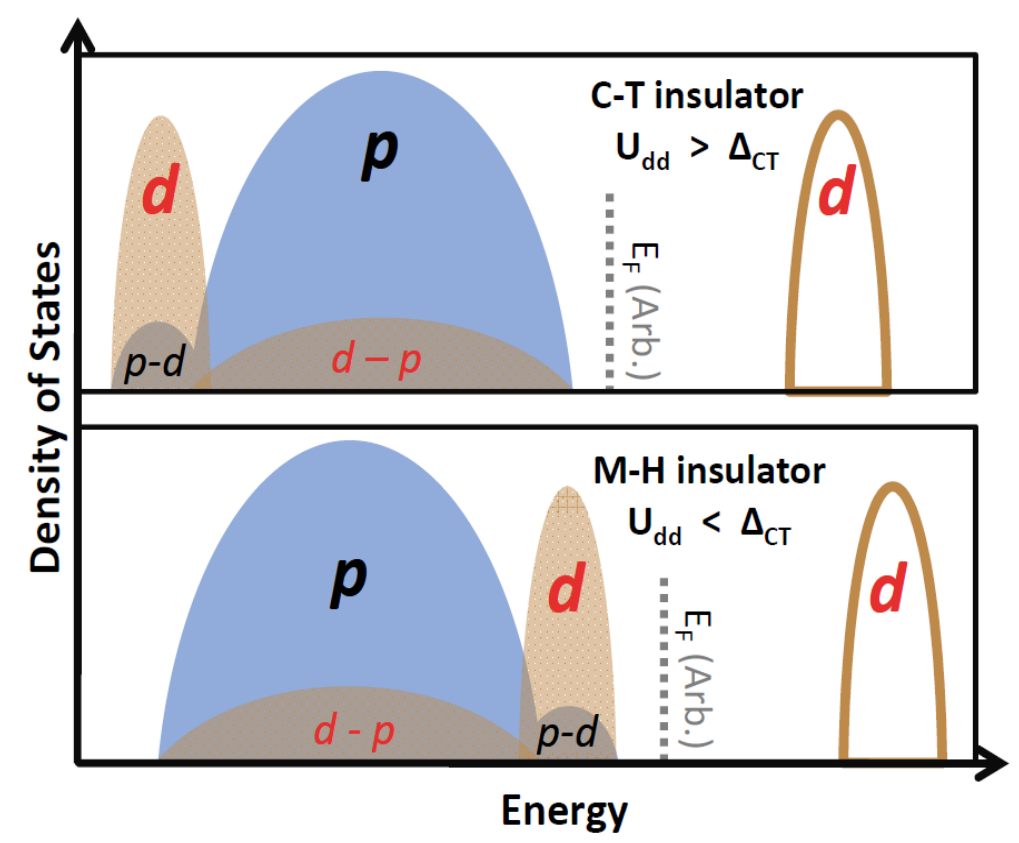


Fig.2

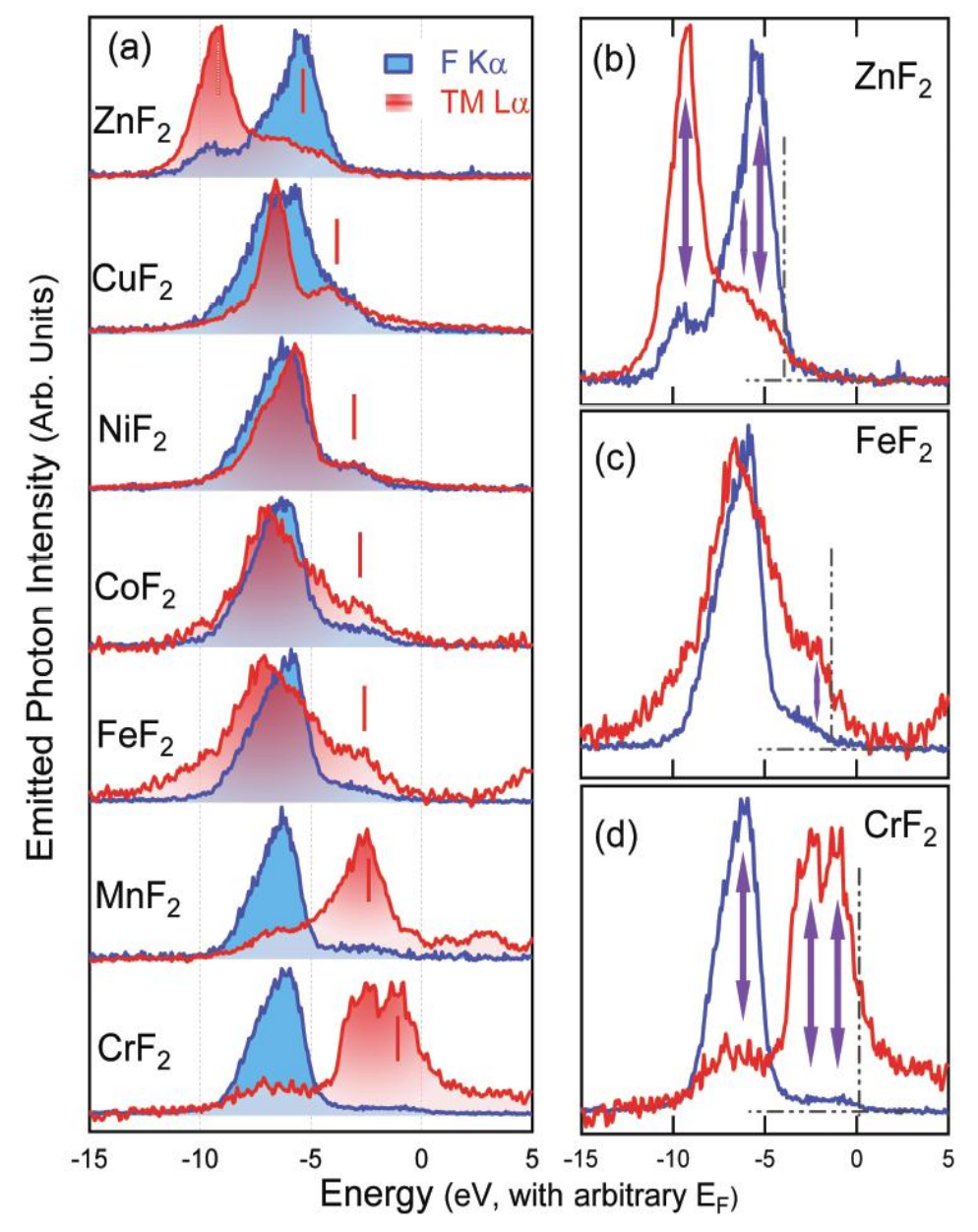


Fig.3

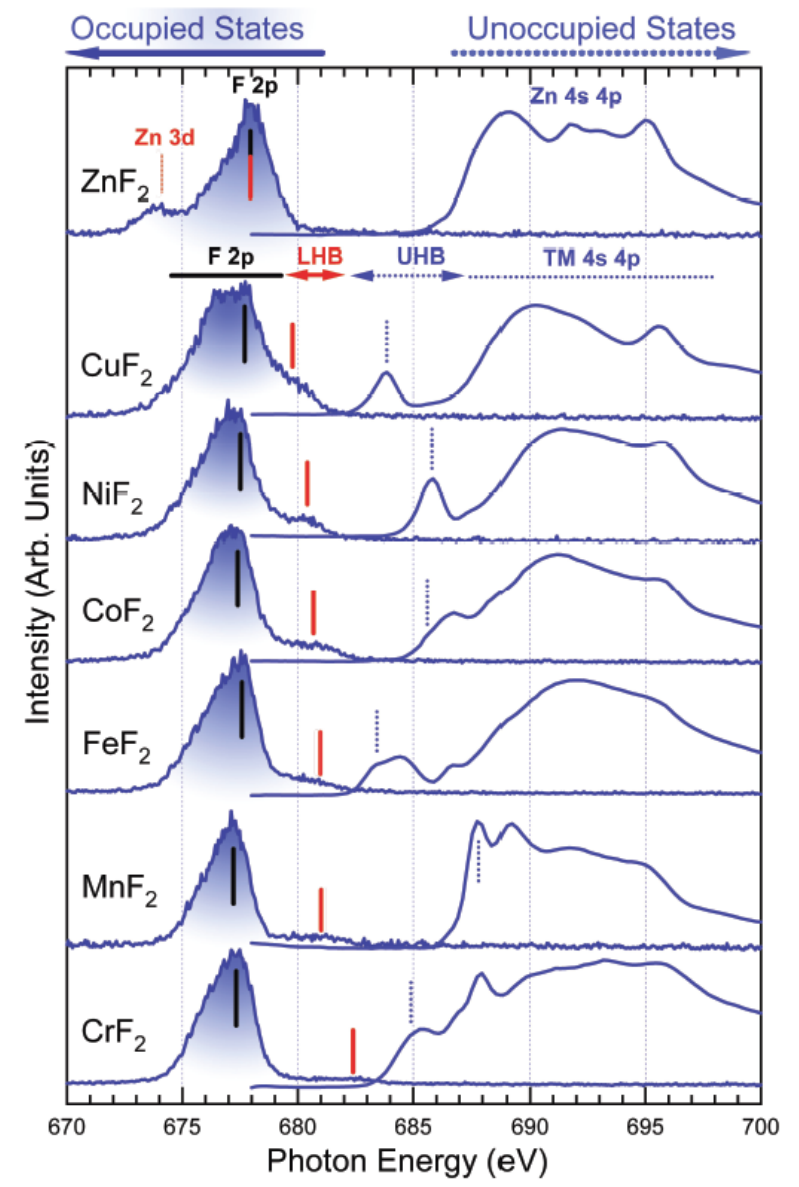


Fig.4

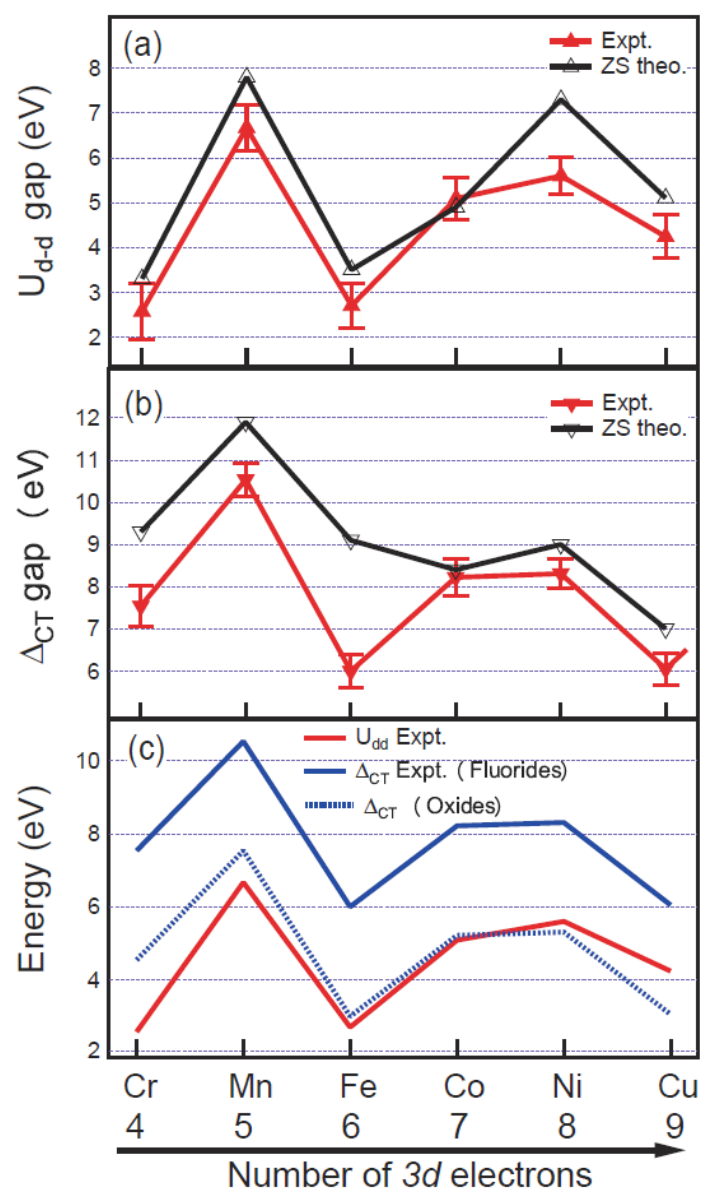




\section{DISCLAIMER}

This document was prepared as an account of work sponsored by the United States Government. While this document is believed to contain correct information, neither the United States Government nor any agency thereof, nor the Regents of the University of California, nor any of their employees, makes any warranty, express or implied, or assumes any legal responsibility for the accuracy, completeness, or usefulness of any information, apparatus, product, or process disclosed, or represents that its use would not infringe privately owned rights. Reference herein to any specific commercial product, process, or service by its trade name, trademark, manufacturer, or otherwise, does not necessarily constitute or imply its endorsement, recommendation, or favoring by the United States Government or any agency thereof, or the Regents of the University of California. The views and opinions of authors expressed herein do not necessarily state or reflect those of the United States Government or any agency thereof or the Regents of the University of California. 